\title{
Influence du procédé de maturation sur les propriétés et la composition des caillés lactiques obtenus par coagulation du lait concentré
}

\author{
par \\ B. TARODO DE LA FUENTE*, S. TURK*, C. ALAIS**, \\ G. HUTIN*** et J.C. BRUNEAU***
}

\section{I. - Introduction}

Les travaux réalisés précédemment sur les caillés acides ont montré que la concentration du lait par évaporation provoquait une légère baisse du pH, qui n'empêchait pas une modification de l'équilibre minéral, avec déplacement de la phase soluble vers la phase colloïdale [1]. Quand l'acidification a lieu, qu'elle soit directe, par addition d'acide chlorhydrique, ou biologique après ensemencement avec des ferments lactiques, elle entraîne la solubilisation des minéraux comme dans un lait normal.

L'acidification du lait par addition d'un acide, à la place de la fermentation lactique, est un procédé de fabrication de caillés ( " direct acid curds ») qui se répand en Amérique du Nord surtout, depuis les travaux d'Ernstrom [2a, 2b]. Récemment, les études de Fox et Ernstrom [2b] ont montré que la caséine ne précipite pas à basse température en présence d'une quantité minimum de calcium et que le refroidissement du lait avant l'acidification ralentissait et limitait la solubilisation du calcium et conduisait, après réchauffage, à des pâtes de plus grande fermeté.

Le but des expériences rapportées dans cet article est de préciser les paramètres technologiques qui interviennent dans la fabrication des pâtes fraîches « $\mathrm{SH}$ » [3] à partir de lait concentré. Nous avons voulu, d'une part, compléter les connaissances déjà acquises sur le lait concentré acidifié normalement et le caillé obtenu [1], en faisant-varier la température du lait maturé et en effectuant l'analyse du sédiment d'ultra-centrifugation et la mesure de l'eau d'hydratation. D'autre part, nous avons cherché à connaître l'influence res-

\footnotetext{
* Ecole Supérieure de Laiterie (ENSAIA), 28 bis, rue Ste-Catherine (54) Nancy.

** Laboratoire de Biochimie appliquée, Université de Nancy I.

*** Service de Recherche des Ets J. Hutin (51) Blaise-sous-Arzillières.
} 
pective des deux modes d'acidification (acidification lactique et acidification directe) sur les propriétés du caillé.

Les fabrications expérimentales ont été effectuées d'octobre 1970 à janvier 1971 dans les Etablissements Jules Hutin (Blaise-sous-Arzillières) par le procédé continu SH-13 ou "Paracurd ".

Dans un prochain article nous discuterons la mesure de l'eau d'hydratation et de l'eau liée déterminée par la méthode cryoscopique.

\section{II. - Matériel et méthodes}

\section{1) LAIT}

Les fabrications ont été réalisées avec du lait écrémé de grand mélange pasteurisé une première fois à $80^{\circ} \mathrm{C}$ pendant $90 \mathrm{~s}$ avant écrémage. A cause d'impératifs technologiques ce lait est stocké de $1 \mathrm{~h}$ à $3 \mathrm{~h}$ à $4-5^{\circ} \mathrm{C}$; il sera de nouveau pasteurisé à $72^{\circ} \mathrm{C}$ pendant $30 \mathrm{~s}$ avant concentration.

L'extrait sec des laits concentrés est voisin de $220 \mathrm{~g}$ par $\mathrm{kg}$, ce qui correspond à une concentration de 2,4 fois environ. Pour certains essais on a ajouté du chlorure de calcium à raison de 4,5 mmoles de calcium par $\mathrm{kg}$ de lait concentré ; pour d'autres, le lait a été conservé à une température de $5^{\circ} \mathrm{C}$ ou $12^{\circ} \mathrm{C}$ pendant $2 \mathrm{~h}$ ou $5 \mathrm{~h}$ après acidification à $\mathrm{pH} 4,8$ et avant coagulation.

2) Maturation biologique

On a utilisé des ferments lactiques «Flora Danica » à raison de $14 \mathrm{~g}$ de ferments pour $100 \mathrm{~g}$ de lait concentré à $220 \mathrm{~g}$ d'extrait sec. La maturation se poursuit pendant $15 \mathrm{~h}$ à $22^{\circ} \mathrm{C}$.

\section{3) Acidification chimioue}

Le lait concentré, ensemencé en ferments lactiques comme précédemment, est immédiatement acidifié à $\mathrm{pH}$ 4,7 à la température de $5^{\circ} \mathrm{C}$ par addition d'acide chlorhydrique à raison de $12,5 \mathrm{~kg}$ d'acide normal pour $100 \mathrm{~kg}$ de lait.

\section{4) Coagulation}

Elle est consécutive au réchauffement à $40^{\circ} \mathrm{C}$ par addition d'eau chaude au lait écrémé concentré maturé, qui se trouve à $22^{\circ} \mathrm{C}$, $12^{\circ} \mathrm{C}$ ou $5^{\circ} \mathrm{C}$. La température de l'eau est déterminée en tenant compte de la quantité de chaleur qu'il faut fournir au lait de départ et du volume qu'il faut lui ajouter pour obtenir un mélange à $40^{\circ} \mathrm{C}$ et à 90 p. 1000 d'extrait sec.

\section{5) MÉthodes analytiques}

Les extraits secs sont déterminés par la méthode au sable et à l'étuve, à $100^{\circ} \mathrm{C} \pm 2^{\circ} \mathrm{C}$.

La fermeté des pâtes est mesurée après $20 \mathrm{~h}$ de conservation à $4^{\circ} \mathrm{C}$ à l'aide du pénétromètre automatique AP $4 / 1$ (Veb-Feinmess, 
TABLEAU 1. - Composition chimique des laits utilisés*

\begin{tabular}{|c|c|c|c|c|c|c|c|c|c|c|c|c|c|c|c|c|c|c|c|c|}
\hline \multirow{2}{*}{$\mathrm{N}^{\circ}$} & \multicolumn{2}{|c|}{1} & \multicolumn{2}{|c|}{2} & \multicolumn{2}{|c|}{3} & \multicolumn{2}{|c|}{4} & \multicolumn{2}{|r|}{5} & \multicolumn{2}{|c|}{6} & \multicolumn{2}{|c|}{7} & \multicolumn{2}{|r|}{8} & \multicolumn{2}{|c|}{9} & \multicolumn{2}{|c|}{10} \\
\hline & LN & LC & LN & LC & LN & LC & $\mathrm{LN}$ & LC & LN & LC & LN & LC & LN & LC & $\mathrm{LN}$ & LC & LN & LC & $\mathrm{LN}$ & LC \\
\hline $\mathrm{pH}$ & 6,62 & 6,52 & 6,65 & 6,52 & 6,48 & 6,42 & 6,55 & 6,40 & 6,70 & 6,55 & 6,52 & 6,32 & 6,52 & 6,35 & 6,52 & 6,42 & 6,48 & 6,35 & 6,62 & 6,54 \\
\hline E.S. $\mathrm{g} / \mathrm{kg}$ & 91,7 & 218 & 91,5 & 208 & 92 & 222 & 91,9 & 221,5 & 83,8 & 214,1 & 90,6 & 227,2 & 93,2 & 227,4 & 89,9 & 217,3 & 89,7 & 218,7 & 87,5 & 217,4 \\
\hline Matières azotées $\mathrm{g} / \mathrm{kg}$ & 33,6 & 80,7 & 33,6 & 78 & 35,4 & 84 & 33,7 & 82,4 & 32,3 & 76 & 34 & 83,5 & 35,7 & 86,9 & 32,7 & 78,5 & 34,3 & 82,4 & 32,7 & 78,2 \\
\hline Facteur de concentration** & & 2,39 & & 2,3 & & 2,39 & & 2,43 & & 2,45 & & 2,48 & & 2,43 & & 2,41 & & 2,42 & & 2,43 \\
\hline Lactose $\mathrm{g} / \mathrm{kg}$ & 46,5 & 109,2 & 46 & 102,1 & 48,1 & 113,3 & 47,6 & 116,5 & 49,1 & 118,4 & 46,5 & 114,1 & 46 & 113,3 & 47,6 & 115,9 & 45,5 & 108,7 & 46 & 112,2 \\
\hline Calcium $\mathrm{g} / \mathrm{kg}$ & 1,26 & 3,13 & 1,3 & 2,99 & 1,14 & 2,9 & 1,35 & 3,22 & 1,24 & 2,81 & 1,2 & 2,92 & 1,28 & 3,14 & 1,28 & 3,01 & 1,2 & 2,97 & 1,24 & 2,9 \\
\hline p. 100 de Ca soluble & 29,4 & 21,2 & 33 & 23,7 & 32,4 & - & 28,6 & 20,2 & 35,5 & 27,2 & 32,5 & 25,2 & 30,9 & 22 & 33,1 & 22,2 & 36,3 & 26,2 & 33,6 & 25,5 \\
\hline Eau d'hydratation $\mathrm{g} / \mathrm{kg}$ & 66,9 & 138 & 79 & 153,5 & 64,9 & 150 & 71,4 & 143,5 & 54,7 & 144,1 & 60,5 & 123 & 66,6 & 149,5 & 64,6 & 143,5 & 64,3 & 148,9 & 60,3 & 146 \\
\hline
\end{tabular}

* Après pasteurisation (L.N.) et concentration (L.C.).

** Calculé d'après l'analyse de l'extrait sec et de l'azote total. 
Dresde) en utilisant le corps plongeant décrit par Schalinatus et Behnke [4] pour la mesure dans les gels mous. Dans les tableaux de résultats, la fermeté est exprimée par la valeur de l'indice de fermeté :

$$
\mathrm{IF}=\frac{1000}{\mathrm{~A}}
$$

A étant la valeur de l'enfoncement du corps plongeant lue directement en dixième de $\mathrm{mm}$ sur l'échelle du pénétromètre. Cet indice croît avec la fermeté ; il est de l'ordre de 20 pour une pâte fraîche normale.

Les matières azotées sont dosées par micro-kjeldahl et le lactose par la méthode de G. Bertrand. Le dosage des minéraux totaux est effectué sur les laits, les sérums et les pâtes ; celui des minéraux solubles est réalisé dans les ultrafiltrats du lait obtenus par la technique de Tessier et Rose [5]. Toutes les analyses sont faites par absorption atomique avec un spectrophotomètre Jobin-Yvon, modèle Delta, après avoir dilué les produits 200 fois et ajouté du chlorure de lanthane comme tampon spectral $[1,6]$. On a tenu compte de la teneur en eau d'hydratation des protéines dans l'expression des résultats des minéraux solubles.

La mesure de l'eau d'hydratation dans les différents laits et pâtes est réalisée par ultra-centrifugation d'après la méthode décrite par Thompson et al. [7, 8]. On utilise la centrifugeuse Spinco L-2, avec une accélération maximum de $68000 \mathrm{xg}(1)$, pendant $35 \mathrm{mn}$ à $37^{\circ} \mathrm{C}$. L'humidité du sédiment correspond à l'eau d'hydratation ; on la calcule par $\mathrm{kg}$ de lait ou de pâte. Il est à noter que l'application d'un champ centrifuge plus élevé ne modifie ni le poids de sédiment, ni la teneur en eau d'hydratation.

\section{III. - Résultats}

A. - EFFETS dE LA CONCENTRATION SUR LA COMPOSITION DU LAIT ET DU SÉDIMENT D'ULTRA-CENTRIFUGATION

\section{A.1. Composition des laits}

Le tableau 1 confirme nos précédents résultats [1] ; il montre une chute du $\mathrm{pH}$ de 0,06 à 0,2 unité pour un taux de concentration calculé à partir des teneurs en extrait sec et en matières azotées de 2,30 à 2,50 . Cette concentration est suffisante pour provoquer une diminution importante du pourcentage de calcium soluble qui passe en moyenne de 32,5 p. 100 dans le lait normal à 22,9 p. 100 dans le lait concentré.

La quantité d'eau d'hydratation trouvée pour le lait écrémé normal varie assez fortement, de 54,7 à $79 \mathrm{~g}$ par $\mathrm{kg}$ autour d'une valeur moyenne égale à $66 \mathrm{~g}$. Après concentration du lait, la teneur moyenne en eau d'hydratation est de $144 \mathrm{~g}$ par $\mathrm{kg}$. Le rapport moyen entre l'eau

(1) Champ centrifuge obtenu avec le rotor Bechman type 50 à $29000 \mathrm{t} / \mathrm{mn}$. 
d'hydratation du lait concentré et celle du lait normal est égal ̀̀ 2,2 ; il est donc légèrement inférieur au rapport de concentration, ce qui pourrait faire penser à un effet déshydratant de la concentration. En effet, l'eau d'hydratation par gramme de sédiment sec est presque toujours moins élevée dans le lait concentré que dans le lait normal ; par contre, par gramme de protéines sédimentées, l'écart entre le lait concentré et le lait normal est faible et de signe variable comme le montre le tableau 2.

\section{A.2. Composition des sédiments d'ultra-centrifugation}

Le poids des sédiments varie peu pour des laits semblables (tab. 2). Pour les laits normaux le poids moyen du sédiment est de $32 \mathrm{~g}$ par $\mathrm{kg}$; pour les laits concentrés, il est égal à $79,4 \mathrm{~g}$. Le rapport moyen entre le poids de sédiment du lait concentré et celui du lait normal est égal à 2,48 ; c'est-à-dire voisin du rapport de concentration calculé d'après la composition des laits.

Le tableau 2 montre, en outre, que la quantité de calcium trouvée dans le sédiment est très variable, aussi bien pour le lait normal que pour le lait concentré. Pour trois laits $\left(n^{o s} 4,5,6\right)$, récoltés au cours de la même période, du 12 au 20 octobre 1970, la proportion de calcium sédimenté et la quantité de calcium liée aux protéines du sédiment sont faibles. Nous ne voyons pas d'explication à ces variations, sinon que la quantité de calcium liée aux protéines sédimentées pourraient être une caractéristique du lait. Cette quantité varie également avec la concentration : les sédiments des laits concentrés sont plus riches en calcium que ceux des laits normaux, ce qui correspond au déplacement de l'équilibre calcique.

Pour le lait normal, les matières azotées sédimentées représentent en moyenne 81,5 p. 100 du poids du sédiment sec ; pour le lait concentré ce pourcentage tombe à $65,6 \mathrm{p}$. 100. Il y a donc augmentation du poids des substances non protéiques sédimentées, dont le calcium. Cette diminution du pourcentage de matières azotées précipitées dans le sédiment entraîne une diminution de l'eau d'hydratation par gramme de matière sèche, mais la quantité d'eau par gramme de protéines sédimentées est du même ordre de grandeur pour les laits normaux ou concentrés, et parfois même légèrement supérieure pour les seconds (tab. 2).

A.3. Influence de l'addition de calcium sur la composition du lait concentré et du sédiment

L'addition de calcium, à raison de 4,5 mmoles par $\mathrm{kg}$ de lait concentré, est faible par rapport aux doses employées en pratique ; elle entraîne cependant une baisse de $\mathrm{pH}$ de l'ordre de 0,07 unité et une augmentation du poids de sédiment et du pourcentage d'azote précipité pour les trois essais mentionnés dans le tableau 3. Il n'y a pas de variation significative de l'eau d'hydratation, du pourcentage de calcium soluble, ni du pourcentage de calcium précipité dans le sédiment. 
TABLEAU 2. - Poids et composition du sédiment*

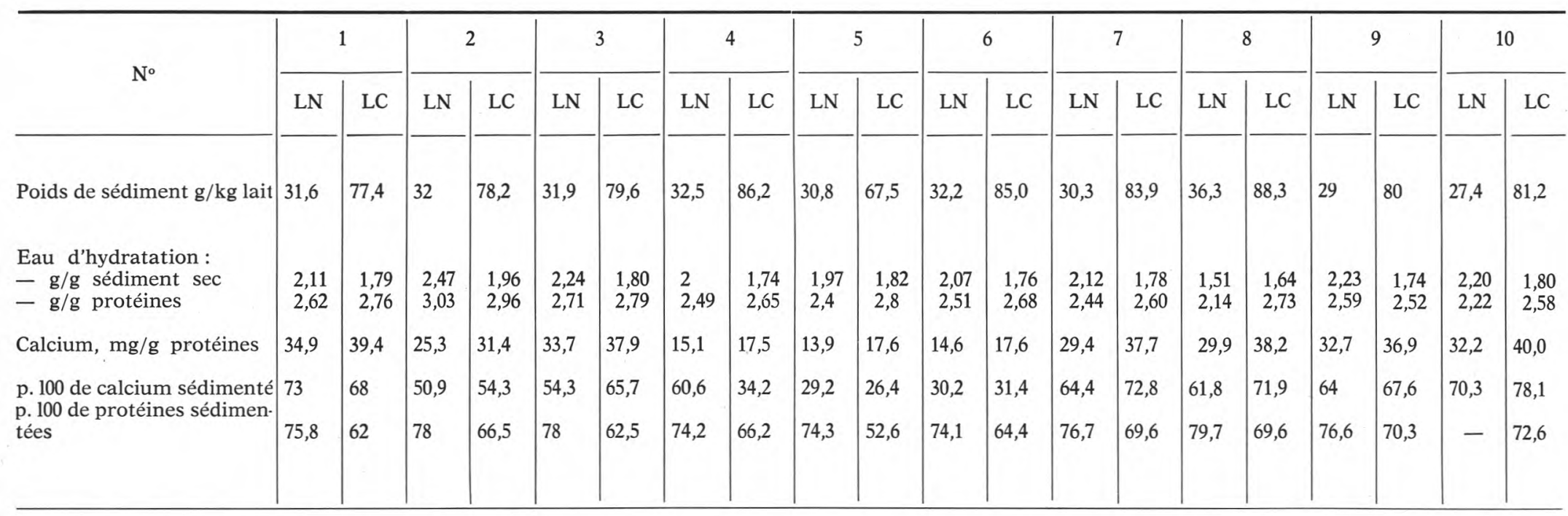

* Sédiments des laits du tableau 1. 
TABLEAU 3. - Influence de l'addition de calcium sur la composition du lait concentré et du sédiment

\begin{tabular}{|c|c|c|c|c|c|c|c|c|c|c|c|c|}
\hline \multirow{2}{*}{$\mathrm{N}^{\circ}$} & \multicolumn{2}{|c|}{$\mathrm{pH}$ du lait } & \multicolumn{2}{|c|}{$\begin{array}{l}\text { Calcium sédimenté } \\
\text { p. } 100 \\
\text { du calcium total }\end{array}$} & \multicolumn{2}{|c|}{$\begin{array}{c}\text { Eau d'hydratation } \\
\text { g/kg de lait }\end{array}$} & \multicolumn{2}{|c|}{$\begin{array}{l}\text { Poids du sédiment } \\
\text { g/kg de lait }\end{array}$} & \multicolumn{2}{|c|}{$\begin{array}{c}\text { Azote du sédiment } \\
\text { p. } 100 \\
\text { de l'azote total }\end{array}$} & \multicolumn{2}{|c|}{$\begin{array}{l}\text { Calcium mg par g } \\
\text { de protéines } \\
\text { sédimentées }\end{array}$} \\
\hline & LC & LC-Ca & LC & LC-Ca & LC & LC-Ca & LC & LC-Ca & LC & LC-Ca & LC & LC-Ca \\
\hline 1 & 6,35 & 6,28 & 67,6 & 64,2 & 139 & 143,5 & 80 & 82,9 & 70,3 & 71,7 & 36,9 & 38,9 \\
\hline 2 & 6,41 & 6,33 & - & - & 149,5 & 148,5 & 85 & 89,5 & 64,4 & 70 & - & - \\
\hline 3 & 6,22 & 6,15 & 72,8 & 72,4 & 148,9 & 149 & 83,9 & 86,3 & 69,6 & 72,5 & 37,7 & 38,1 \\
\hline
\end{tabular}

LC : Lait concentré.

LC-Ca : Lait concentré additionné de calcium. 
B. - Conséquences technologiques du type de maturation et de La CONSERVATION DU LAIT CONCENTRÉ MATURÉ

B.1. Effets de l'addition de calcium et de la température du lait au moment de la coagulation

Des expériences ont été réalisées à trois températures du lait acidifié : $2^{\circ} \mathrm{C}, 12^{\circ} \mathrm{C}$ et $22^{\circ} \mathrm{C}$, avant la coagulation à $40^{\circ} \mathrm{C}$ par addition d'eau chaude. Avec l'acidification directe, l'essai à $22^{\circ} \mathrm{C}$ n'est pas possible car le lait ne peut être pompé dans l'appareil ; en effet, la caséine a floculé ; alors qu'avec l'acidification lactique on obtient un produit homogène à cette température. Dans ces expériences l'extrait sec du lait maturé et son $\mathrm{pH}$ (autour de 4,8) ne varient pratiquement pas. De plus, on a fait une série parallèle pour apprécier l'effet d'une addition de chlorure de calcium.

Le tableau 4 montre que la température du lait acidifié n'a pas d'influence sur l'extrait sec du caillé et que son effet sur la fermeté n'est pas significatif dans le cas de la maturation lactique. Dans le cas de l'acidification chimique, la température de $12^{\circ} \mathrm{C}$ donne des indices de fermeté plus élevés.

En ce qui concerne le sédiment (obtenu à $68000 \mathrm{xg}$ ), l'eau d'hydratation varie très peu avec la température. Par contre, le pourcentage des protéines sédimentées, dans le cas de l'acidification lactique, augmente sensiblement lorsque la température du lait maturé décroît ; alors qu'il varie peu dans le cas de l'acidification chimique.

Avec le caillé formé par acidification lactique, on observe un effet étonnant de l'addition de chlorure de calcium : la fermeté diminue ; à $22^{\circ} \mathrm{C}$ et $12^{\circ} \mathrm{C}$ le caillé devient fluide. Avec l'acidification directe, on constate, au contraire, que l'enrichissement en calcium entraîne un accroissement de la fermeté ; notamment à $12^{\circ} \mathrm{C}$, accompagné d'une légère diminution de l'eau d'hydratation.

Il est important de noter que les rendements obtenus par acidj. fication chimique sont toujours très inférieurs aux rendements obtenus par fermentation lactique, alors que les extraits secs de la pâte sont beaucoup plus élevés dans le premier cas que dans le second. En outre le dosage du lactose (qui n'est pas reporté dans le tableau 4) montre que le caillé lactique en contient nettement plus (en moyenne 31 p. 100 de l'extrait sec) que le caillé à l'acide chlorhydrique (23 p. 100).

Les résultats du tableau 4 et ceux qui seront exposés plus loin montrent qu'il n'y a pas de relation entre la fermeté et l'eau d'hydratation des protéines, déterminée par sédimentation. Par contre, il existe une corrélation entre la fermeté et ce que nous avons désigné sous le terme " rapport des extraits secs »; c'est-à-dire le rapport qui existe entre l'extrait sec apporté par le sérum dans la pâte et l'extrait sec restant, constitué pour la plus grande partie par la caséine précipitée. Lorsqu'il dépasse 0,9 pour les expériences effec- 


\begin{tabular}{|c|c|c|c|c|c|c|c|c|c|c|c|c|c|}
\hline \multirow{3}{*}{$\begin{array}{c}\text { Température } \\
\text { du lait }\end{array}$} & \multirow{3}{*}{$\begin{array}{l}\mathrm{Ca} \\
(2)\end{array}$} & \multicolumn{8}{|c|}{ Caillé } & \multicolumn{4}{|c|}{ Sédiment } \\
\hline & & \multicolumn{2}{|c|}{$\begin{array}{l}\text { Extrait sec } \\
\text { p. } 100\end{array}$} & \multicolumn{2}{|c|}{$\begin{array}{c}\text { Rapport des ES } \\
\text { (3) }\end{array}$} & \multicolumn{2}{|c|}{$\begin{array}{c}\text { Fermeté } \\
\text { (4) }\end{array}$} & \multicolumn{2}{|c|}{$\begin{array}{l}\text { Rendement } \\
\text { (5) }\end{array}$} & \multicolumn{2}{|c|}{$\begin{array}{c}\text { Eau d'hydrata- } \\
\text { tion }(\mathrm{g} / \mathrm{g}) \\
\text { protéines } \\
\text { sédimentées }\end{array}$} & \multicolumn{2}{|c|}{$\begin{array}{c}\text { p. } 100 \text { de } \\
\text { protéines } \\
\text { sédimentées }\end{array}$} \\
\hline & & FL & $\mathrm{AC}$ & FL & $\mathrm{AC}$ & FL & AC & FL & $\mathrm{AC}$ & FL & $\mathrm{AC}$ & FL & $\mathrm{AC}$ \\
\hline $22^{\circ} \mathrm{C}$ & $\begin{array}{l}0 \\
+\end{array}$ & $\begin{array}{l}14 \\
12,5\end{array}$ & & $\begin{array}{l}0,76 \\
1\end{array}$ & & $\begin{array}{l}15 \\
<3\end{array}$ & & $\begin{array}{l}54,2 \\
55\end{array}$ & & $\begin{array}{l}1,5 \\
1,4\end{array}$ & & $\begin{array}{l}90,5 \\
83,4\end{array}$ & \\
\hline $12^{\circ} \mathrm{C}$ & $\begin{array}{l}0 \\
+\end{array}$ & $\begin{array}{l}14,4 \\
11,9\end{array}$ & $\begin{array}{l}19,6 \\
19,1\end{array}$ & $\begin{array}{l}0,69 \\
1,13\end{array}$ & $\begin{array}{l}0,34 \\
0,39\end{array}$ & $\begin{array}{r}20 \\
<3\end{array}$ & $\begin{array}{r}125 \\
>300\end{array}$ & $\begin{array}{l}57,1 \\
53,7\end{array}$ & $\begin{array}{l}39,4 \\
35\end{array}$ & $\begin{array}{l}1,4 \\
1,4\end{array}$ & $\begin{array}{l}1,4 \\
1,2\end{array}$ & $\begin{array}{l}94,7 \\
87\end{array}$ & $\begin{array}{l}96 \\
96,6\end{array}$ \\
\hline $2^{\circ} \mathrm{C}$ & $\begin{array}{l}0 \\
+\end{array}$ & $\begin{array}{l}14,3 \\
14\end{array}$ & $\begin{array}{l}18,3 \\
19,1\end{array}$ & $\begin{array}{l}0,68 \\
0,72\end{array}$ & $\begin{array}{l}0,41 \\
0,39\end{array}$ & $\begin{array}{r}21 \\
7\end{array}$ & $\begin{array}{l}58 \\
71\end{array}$ & $\begin{array}{l}54 \\
53\end{array}$ & $\begin{array}{l}36,2 \\
33,4\end{array}$ & $\begin{array}{l}1,5 \\
1,6\end{array}$ & $\begin{array}{l}1,35 \\
1,2\end{array}$ & $\begin{array}{l}97 \\
94\end{array}$ & $\begin{array}{c}96,2 \\
95,9\end{array}$ \\
\hline
\end{tabular}

(1) FL : Maturation par addition de ferments lactiques ; AC : acidification chimique.

(2) Le signe + indique une addition de 4,5 mmoles de calcium par $\mathrm{kg}$ de lait concentré $\left(0,5 \mathrm{~g} \mathrm{CaCl}_{2} / \mathrm{kg}\right)$.

(3) Extrait sec apporté par le sérum dans le caillé/extrait sec restant.

(4) Le chiffre $<3$ correspond à un produit très fluide ) Le chiffre $>300$ correspond à un produit très ferme

mesure impossible avec le système utilisé.

(5) Pourcentage de matière sèche du caillé par rapport à celle du lait concentré mis en œuvre. 
TABLEAU 5. - Effets du temps de conservation sur la composition et les caractéristiques des caillés et des sédiments*

\begin{tabular}{|c|c|c|c|c|c|c|c|c|c|c|c|c|c|c|}
\hline \multirow{3}{*}{$\begin{array}{c}\text { Température } \\
\text { du lait }\end{array}$} & \multirow{3}{*}{$\begin{array}{c}\text { Durée de } \\
\text { conservation }\end{array}$} & \multirow{3}{*}{$\mathrm{Ca}$} & \multicolumn{8}{|c|}{ Caillé } & \multicolumn{4}{|c|}{$\begin{array}{c}\text { Sédiment } \\
\text { (par } \mathrm{g} \text { de protéines) }\end{array}$} \\
\hline & & & \multicolumn{2}{|c|}{$\begin{array}{l}\text { Extrait sec } \\
\text { p. } 100\end{array}$} & \multicolumn{2}{|c|}{$\begin{array}{l}\text { Rapport } \\
\text { des E.S. }\end{array}$} & \multicolumn{2}{|c|}{ Fermeté } & \multicolumn{2}{|c|}{$\begin{array}{l}\text { Rendement } \\
\text { en E.S. }\end{array}$} & \multicolumn{2}{|c|}{$\begin{array}{l}\text { Calcium } \\
(\mathrm{mg})\end{array}$} & \multicolumn{2}{|c|}{$\underset{\text { Eau }}{\text { d'hydratation }}$} \\
\hline & & & FL & $\mathrm{AC}$ & FL & $\mathrm{AC}$ & FL & $\mathrm{AC}$ & FL & $\mathrm{AC}$ & $\mathrm{FL}$ & AC & FL & $\mathrm{AC}$ \\
\hline \multirow{3}{*}{$12^{\circ} \mathrm{C}$} & 0 & $\begin{array}{l}0 \\
+\end{array}$ & $\begin{array}{l}13,2 \\
14,3\end{array}$ & $\begin{array}{l}19,6 \\
19,1\end{array}$ & $\begin{array}{l}0,77 \\
0,6\end{array}$ & $\begin{array}{l}0,34 \\
0,39\end{array}$ & $\begin{array}{l}20,8 \\
41,5\end{array}$ & $\begin{array}{r}125 \\
>300\end{array}$ & $\begin{array}{l}50,8 \\
50,3\end{array}$ & $\begin{array}{l}39,5 \\
35\end{array}$ & $\begin{array}{l}2,7 \\
2,6\end{array}$ & $\begin{array}{l}2,6 \\
4,4\end{array}$ & $\begin{array}{l}1,5 \\
1,5\end{array}$ & $\begin{array}{l}1,4 \\
1,2\end{array}$ \\
\hline & $2 \mathrm{~h}$ & $\begin{array}{l}0 \\
+\end{array}$ & $\begin{array}{l}13,7 \\
13,3\end{array}$ & $\begin{array}{l}16,9 \\
19,1\end{array}$ & $\begin{array}{l}0,88 \\
0,75\end{array}$ & $\begin{array}{l}0,45 \\
0,38\end{array}$ & $\begin{array}{l}23,8 \\
20,4\end{array}$ & $\begin{array}{l}45 \\
67\end{array}$ & $\begin{array}{l}53 \\
51\end{array}$ & $\begin{array}{l}43 \\
35,6\end{array}$ & $\begin{array}{l}2,7 \\
2,5\end{array}$ & $\begin{array}{l}3 \\
5,1\end{array}$ & $\begin{array}{l}1,5 \\
1,6\end{array}$ & $\begin{array}{l}1,5 \\
1,3\end{array}$ \\
\hline & $5 \mathrm{~h}$ & $\begin{array}{l}0 \\
+\end{array}$ & $\begin{array}{l}12,1 \\
13,5\end{array}$ & $\begin{array}{l}15,9 \\
18,3\end{array}$ & $\begin{array}{l}0,92 \\
0,79\end{array}$ & $\begin{array}{l}0,47 \\
0,42\end{array}$ & $<_{16,1}^{3}$ & $\begin{array}{l}23 \\
77\end{array}$ & $\begin{array}{l}67,3 \\
52,3\end{array}$ & $\begin{array}{l}43,5 \\
38,6\end{array}$ & $\begin{array}{l}2,2 \\
2,1\end{array}$ & $\begin{array}{l}2,4 \\
5\end{array}$ & $\begin{array}{l}1,5 \\
1,6\end{array}$ & $\begin{array}{l}1,4 \\
1,3\end{array}$ \\
\hline
\end{tabular}

* Les symboles et abréviations sont les mêmes que dans le tableau 4. 
tuées, les caillés obtenus sont liquides ; au-dessous de 0,9 , ils sont d'autant plus fermes qu'il est plus bas.

B.2. Effets du temps de stockage à une température donnée sur la fabrication

La conservation à $12^{\circ} \mathrm{C}$ du lait acidifié naturellement ou par addition d'acide, avant la coagulation, provoque une diminution de l'extrait sec du caillé et de sa fermeté comme le montre le tableau 5. On observe corrélativement une augmentation du rapport des extraits secs (la proportion d'extrait sec de sérum augmente dans le caillé); par contre, l'eau d'hydratation exprimée par rapport aux protéines sédimentées varie très peu. Il en est de même de la quantité de calcium sédimentée par gramme de protéines.

L'augmentation du temps de conservation accroît le rendement en extrait sec et, comme dans les expériences précédentes, l'acidification directe donne des caillés bien plus fermes et avec des extraits sec élevés (écart de 6 p. 100 environ), mais l'abaissement du rendement est très fort (de 10 à 20 p. 100 environ).

Lorsque le lait est additionné de chlorure de calcium, le tableau 5 montre que les résultats varient dans le même sens que les précédents, mais plus faiblement. Il faut noter, cependant, dans le cas d'acidification directe, que lorsque la quantité de calcium est élevée dans le sédiment, il se produit généralement une baisse de l'eau d'hydratation. D'autre part, l'influence du temps de conservation sur la fermeté et le rapport des extraits secs est diminuée.

Avec le lait utilisé dans ces expériences, l'addition de calcium n'a pas provoqué la fluidification que l'on avait observé à $12^{\circ} \mathrm{C}$ et à $22^{\circ} \mathrm{C}$ dans les expériences précédentes (tab. 4) pour la maturation lactique.

Deux autres séries d'essais parallèles réalisées à $5^{\circ} \mathrm{C}$ après maturation biologique ont montré une évolution dans le même sens de tous les paramètres.

\section{IV. - Discussion}

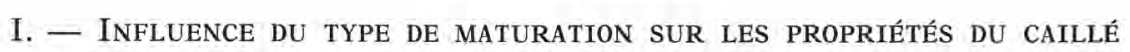
ET LE RENDEMENT

Les résultats obtenus à partir d'une maturation lactique (pâtes fraîches classiques) ou d'une acidification chimique permettent d'établir une comparaison entre les deux types de caillé, qui est schématiquement présentée dans le tableau 6 .

Les extraits secs des fabrications réalisées par acidification chimique sont plus élevés que ceux des caillés classiques. Ils pourraient être abaissés par le choix de paramètres technologiques différents, mais dans ce cas, la fermeté des caillés correspondants serait certainement diminuée. 
TABLEAU 6. - Caractéristiques des caillés lactiques et chimiques

\begin{tabular}{|c|c|c|}
\hline & $\begin{array}{l}\text { Acidification biologique } \\
\text { (ferments lactiques) }\end{array}$ & $\begin{array}{l}\text { Acidification chimique } \\
\text { (acide chlorhydrique) }\end{array}$ \\
\hline $\begin{array}{l}\text { Extrait sec moyen, g/kg } \\
\text { Lactose, p. } 100 \text { d'E.S. } \\
\text { Calcium, mg par g de protéi- } \\
\text { nes sédimentées } \\
\text { Eau d'hydratation, g par g de } \\
\text { protéines sédimentées } \\
\text { Rendement en extrait sec } \\
\text { Rapport des extraits secs } \\
\text { Fermeté moyenne }\end{array}$ & $\begin{array}{c}128 \\
31,2 \\
2,5 \\
1,5 \\
53,5 \\
0,6 \text { à 2,76 } \\
\text { Faible } \\
\text { (indice inférieur à 20) }\end{array}$ & $\begin{array}{c}176 \\
22,8 \\
3,2 \\
1,3 \\
42,2 \\
0,34 \text { à } 0,47 \\
\text { Elevée } \\
\text { (indice supérieur à 20) }\end{array}$ \\
\hline
\end{tabular}

Le pourcentage de lactose dans l'extrait sec est nettement abaissé par l'acidification directe alors que le taux de calcium trouvé dans le sédiment est au contraire augmenté. L'acidification rapide réalisée à basse température permet d'obtenir une minéralisation importante dans le caillé, comme l'ont montré Fox et Ernstrom [2] : elle concourt, avec la diminution de la teneur en lactose, à une plus grande fermeté du produit. La teneur en eau d'hydratation par gramme de protéines, plus faible que dans les pâtes classiques, intervient sans doute dans le même sens en permettant une meilleure cohésion des matières solides.

Le paramètre le plus significatif, en ce qui concerne la fermeté du caillé, est le "rapport des extraits secs ». Si ce rapport est supérieur à 0,9 dans les conditions de nos essais, les caillés obtenus sont liquides. Ces observations confirment les précédentes [1] qui avaient montré l'importance de la teneur en lactose et de la quantité de calcium fixée dans le caillé sur sa fermeté.

L'acidification chimique directe favorise l'obtention d'un caillé mieux égoutté possédant une plus grande fermeté. Par contre, le poids de caillé obtenu, et surtout la quantité totale de matière sèche recueillie, sont plus faibles. L'acidification chimique conduit donc à des rendements inférieurs.

L'augmentation du temps de conservation du lait entre l'acidification et la coagulation diminue la fermeté des caillés comme l'ont montré Fox et Ernstrom [2]. L'hypothèse de ces auteurs, selon laquelle le calcium serait refixé à la caséine après solubilisation par acidification et coagulation, semble pouvoir être retenue. L'analyse a montré en effet qu'un lait maturé de pH 4,7-4,8 ne contient plus de calcium sous forme insoluble.

L'addition au lait concentré, avant la maturation lactique, de quantités limitées de chlorure de calcium, a eu des effets variables sur la fermeté et l'extrait sec des caillés. D'autres essais seront 
nécessaires pour expliquer les causes de ces variations. Par contre, dans le cas de l'acidification directe, on observe régulièrement après addition de chlorure de calcium, une augmentation de la fermeté et de l'extrait sec des caillés, ainsi que de la teneur en calcium du sédiment, bien que le $\mathrm{pH}$ atteint soit voisin de 4,8 . Il est certain que le calcium joue un rôle dans la coagulation en continu du lait concentré acidifié par l'un ou l'autre type de maturation. Cette constatation est également en faveur de l'hypothèse précédente.

\section{II. - Composition des SÉdiments. EAU D'HYdRATATION}

La cause de la diminution du pourcentage d'azote sédimenté par centrifugation dans le lait concentré par rapport au lait normal, ne se voit pas clairement. Elle peut être liée à une variation de la taille des micelles et à un accroissement de la viscosité du produit. La formation d'un complexe entre la $\beta$ lactoglobuline et la caséine $\chi$, favorisée par la double pasteurisation que subit ce lait, peut également intervenir [9].

La quantité de calcium par gramme de protéines sédimentée augmente avec la concentration du lait mais les valeurs trouvées soni variables, ce qui laisse supposer que d'autres facteurs interviennent sur la fixation du phosphate de calcium par la caséine.

Par ultra-centrifugation, nous avons obtenu une valeur moyenne de 6,6 p. 100 pour l'eau d'hydratation du lait normal, alors que par la méthode cryoscopique, nous avons trouvé 4,0 à 4,5 p. 100 d'eau liée (résultats non encore publiés) et que d'autres auteurs ont trouvé, pour cette même eau liée, une valeur moyenne de 2,2 p. 100 [10]. L'eau d'hydratation doit donc être constituée de l'eau liée telle qu'on la détermine par cryoscopie et d'une certaine quantité d'eau supplémentaire constituée par exemple par la deuxième couche du modèle à trois couches défini par Drost-Hansen [11].

Dans les caillés acides, l'eau d'hydratation est réduite, puisqu'elle n'atteint que 1,2 à 1,6 g par gramme de protéines sédimentées, contre 2 à 3 g dans le lait. Cette diminution est une conséquence de l'acidification jusqu'au point isoélectrique de la caséine, mais elle se produit différemment suivant le type d'acidification : les caillés obtenus par maturation biologique restent plus hydratés (1,5 g par gramme de protéines) que les caillés obtenus par acidification chimique $(1,3 \mathrm{~g}$ par gramme de protéines).

\section{Remerciements}

Nous remercions MM. J. Chanet et J. Tasky et Mlle B. Masson pour leur efficace collaboration.

\section{$R$ és u m é}

La composition et les propriétés des caillés lactiques obtenus par coagulation continue du lait concentré dépendent beaucoup du 
procédé d'acidification utilisé. Les caillés obtenus par acidification directe ont un extrait sec, un taux de calcium fixé et une fermeté supérieure à ceux d'un caillé lactique normal. Ils contiennent par contre moins de lactose et ont un taux d'hydratation plus faible. Le rendement en pâte fraîche est sensiblement diminué avec l'acidification directe. Pour les deux types de caillés, l'allongement du temps de conservation diminue l'extrait sec et la fermeté.

L'ultra-centrifugation à $68000 \mathrm{xg}$ est une méthode simple et reproductible qui permet de déterminer l'hydratation et la minéralisation des protéines d'un lait ou d'un caillé.

Il n'y a pas de relation nette entre la fermeté du caillé et la teneur en eau d'hydratation des protéines. Par contre, il existe une corrélation entre la fermeté et la proportion d'extrait sec de lactosérum restant dans le caillé.

\section{S u $\mathrm{m} \mathrm{m}$ a ry}

The composition and properties of acid curds obtained by continuous coagulation of concentrated milk markedly depend on the acidification process. Direct acid curds contain more total solids and backfixed calcium and less lactose than normal lactic curds; the firmness is higher but the curd yield is lower. The increase of storage period before curdling decreases total solids content and firmness of both curds.

The ultracentrifugation at $68000 \mathrm{xg}$ is a simple and reliable method to determine solvation and mineralisation of milk and curd proteins.

The curd firmness is not correlated to solvation rate but to whey solids content of the curd.

Reçu pour publication en août 1971.

\section{Bibliographie}

[1] Alais (C.) and TARodo DE LA Fuente (B.) (1970). - Effects of milk concentration on the salts equilibria and on the composition and properties of lactic curds. Milchwissenschaft, 25, 514.

[2a] ERnstrom (C. A.) (1965), - Continuous cottage cheese manufacture without culture. Milk Ind., 56, 36.

[2b] Fox (C. W.) and ERnstrom (C. A.) (1969). - The role of calcium in the low-temperature acidification of skim milk and the formation of direct acid curd, J. Dairy Sci., 52, 885.

[3] BRevets S.E.F.F.A.C. Paris (1964).

[4] Schalinatus (E.) und BehnKe (U.) (1969). - Untersuchungen zur Wirkung von Labpraparaten verschiedener Herkunft. 2. Festigkeit und Schrumpfung des Bruches. Milchwissenschaft, 22, 601.

[5] Tessier (H.) and Dyson Rose (1958). - Calcium ion concentration in milk. J. Dairy Sci, 41, 351. 
[6] Linden G. (1971). - Applications de la spectrophotométrie d'absorption atomique dans les laboratoires d'industrie alimentaire. Ind. alim. agric., $88,793$.

[7] Thompson (M. P.), Boswell (R. T.) and Martin (V.) (1969). - Casein-Pellet solvation and Heat stability of individual cow's milk. J. Dairy Sci., 52, 796.

[8] Thompson (M. P.), Gordon (W. G.), Boswell (R. T.) and Farrell (H. M.) (1969). - Solubility, solvation and stabilization of $\alpha_{\mathrm{S} 1}$ and $\beta$ caseins. J. Dairy Sci., 52, 1166.

[9] Della Monica (E. S.), Custer (J. H.) and Zittle (C. A.) (1958). - Effect of calcium chloride and heat on solutions of mixtures of $\beta$-lactoglobulin and casein. J. Dairy Sci., 41, 465.

[10] Pyenson (H.) and Dahle (C. D.) (1938). - Bound water and its relation to some dairy products. J. Dairy Sci., 21, 169.

[11] Drost Hansen (W.) (1969). - Structure of water near solid interfaces. Industrial and Engineering Chemistry, 61, 10. 